\title{
Concevoir une bonne alimentation
}

Les pratiques alimentaires des expéditeurs polaires

Pascal Lièvre, Géraldine Rix-Lièvre et Michel Récopé

\section{(2) OpenEdition}

Journals

Édition électronique

URL : https://journals.openedition.org/tc/8886

DOI : $10.4000 /$ tc. 8886

ISSN : 1952-420X

Éditeur

Éditions de l'EHESS

\section{Édition imprimée}

Date de publication : 17 mai 2018

Pagination : 104-107

ISBN : 978-2-7132-2750-9

ISSN : 0248-6016

Référence électronique

Pascal Lièvre, Géraldine Rix-Lièvre et Michel Récopé, "Concevoir une bonne alimentation », Techniques \& Culture [En ligne], 69 | 2018, mis en ligne le 17 mai 2020, consulté le 29 septembre 2022. URL : http:// journals.openedition.org/tc/8886 ; DOI : https://doi.org/10.4000/tc.8886 


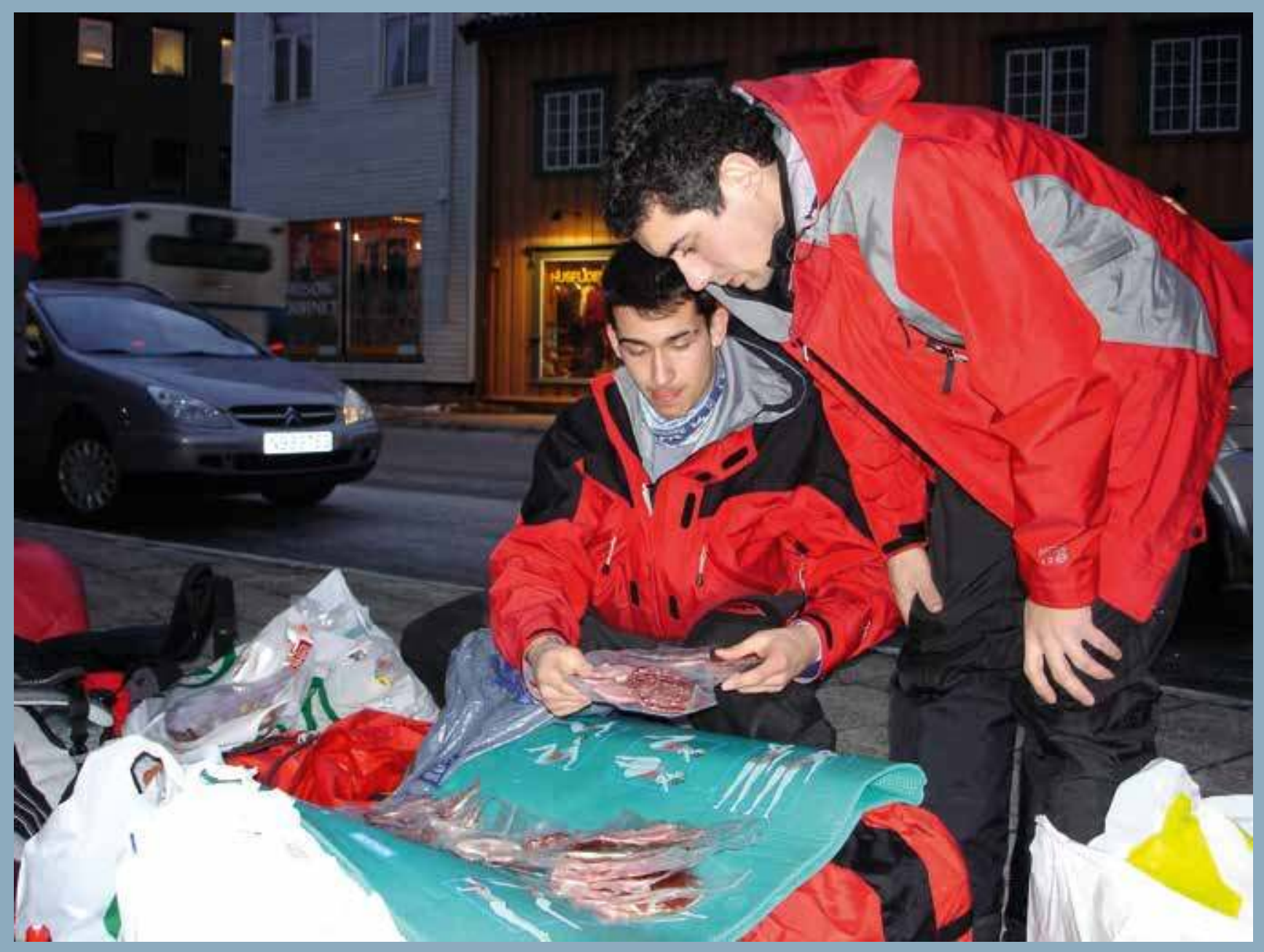




\title{
Concevoir une bonne alimentation
}

Les pratiques alimentaires des expéditeurs polaires

\begin{abstract}
«Départ pour le Groenland dans quelques jours... Première observation participante d'une expédition polaire à ski et premier départ pour le Grand Nord: beaucoup d'excitation mais il me reste encore de nombreux préparatifs à finaliser et quelques interrogations à trancher. Côté matériel technique, tout était au point: ce que je devais apporter pour moi et pour le collectif et la manière dont chaque élément serait embarqué dans l'avion. Restait la nourriture. Les membres de l'expédition avaient choisi d'individualiser les rations: "Comme tout le monde n'aime pas les mêmes choses, il vaut mieux que chacun gère sa nourriture." Il me fallait donc préparer "mes rations" pour 15 jours. Le chef d'expédition m'avait fourni une feuille de calcul indiquant, en fonction de mon poids, le nombre de calories par jour à prévoir. Je devais préparer trois sachets par jour, un pour le matin, un pour les vivres de courses, un pour le soir et surtout contrôler le poids de ma ration journalière pour qu'il ne dépasse pas $1 \mathrm{~kg}$. L'équation pouvait paraître simple, mais sachant que je ne me passe que difficilement de fruits et que je ne prends ni lait, ni matière grasse le matin... Sur ma feuille de calcul, il manquait des calories et le poids était trop important. Pour réduire le poids, je me suis résolue à n'emporter que $50 \mathrm{~g}$ de fruits secs ou en compote par jour et à remplacer les 50 autres grammes par des oléagineux beaucoup plus caloriques. Pour augmenter les calories du matin, j’avais aussi trouvé une préparation en poudre de crème pâtissière à laquelle il fallait ajouter de l'eau. J'avais pris la précaution de la goûter à la maison comme je l'avais fait pour les repas lyophilisés. Malgré les réajustements en fromage et charcuterie, il manquait toujours des calories. Pour résoudre l'équation, je décidais de compléter ma ration journalière par $40 \mathrm{~g}$ de foie gras. Voilà le tour était joué, sur un peu de pain d'épice ou dans la purée du soir, j'allais me régaler... Me régaler, oui le premier soir. Dès le second soir, je proposais à mes coéquipiers un peu de mon repas; dès le quatrième soir, je ne pouvais plus manger le foie gras. Trop gras, le foie gras tous les jours!!! 190 calories par jour non consommées... devenues inconsommables, mais toujours transportées. Mais mes déboires ne s'arrêtaient pas là. Impossible de "cuire" la préparation de crème
\end{abstract}


1 \& 2. Terre de Baffin, 2005

Se nourrir pour progresser... S'arrêter pour profiter d'une collation ensemble... Une question de style?
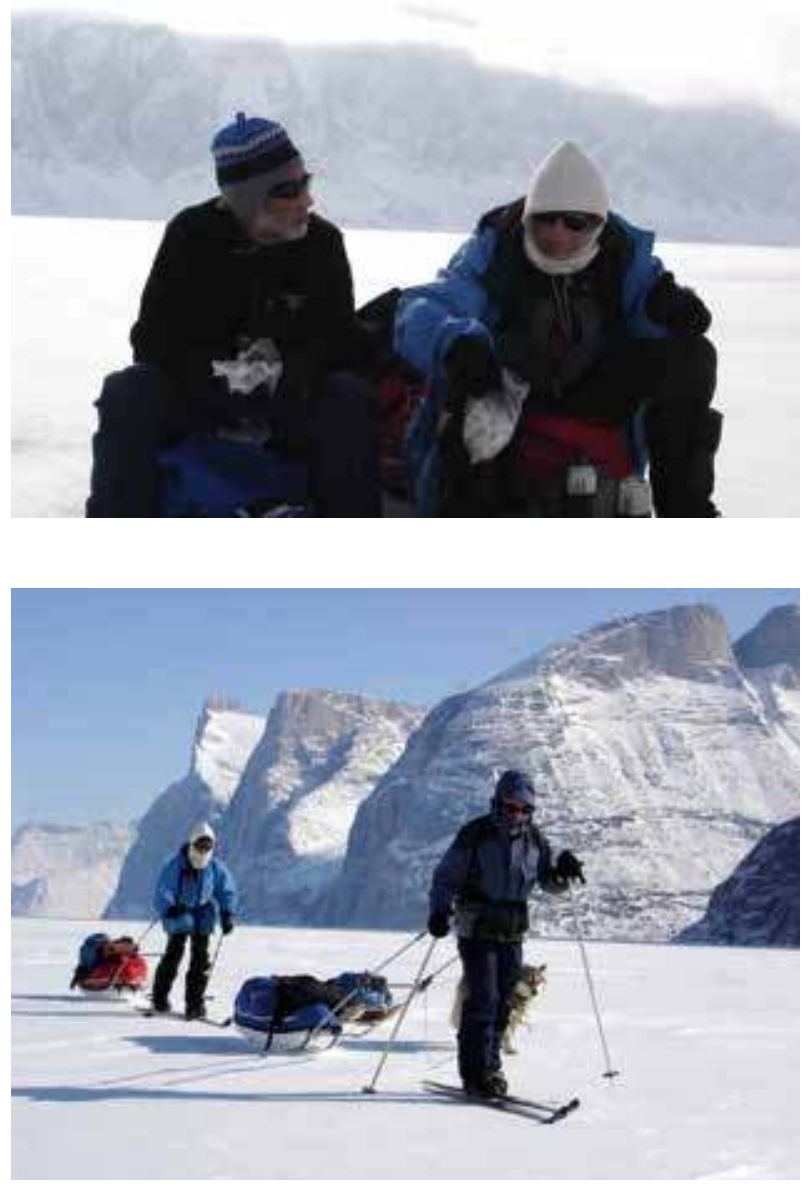

pâtissière: l'eau bouillante ne restait pas assez chaude assez longtemps... encore 180 calories de moins. Au bout de quelques jours, je consommais environ $10 \%$ de calories en moins par rapport à ce que j’avais anticipé, je mangeais mes céréales "à sec" comme des graines. Par contre, de fait, je continuais à porter le poids du foie gras et de la préparation de crème qui s'accumulaient jour après jour dans ma pulka [sorte de luge utilisée pour transporter le matériel au cours de l'expédition et traînée par chaque expéditeur] (Géraldine, Groenland 2003). Ce récit intègre des extraits de journal de bord et des éléments d'analyse. Il montre la manière dont un des coauteurs de cet article a été subjectivement confronté aux questions alimentaires en mettant en lien le temps de la préparation et celui de la consommation. Il souligne la complexité de la question alimentaire. Comment prévoir nos besoins alimentaires et nos goûts dans un contexte en rupture avec notre quotidien? Nous envisageons cette question dans le cadre d'un programme de recherche de management des situations extrêmes (Lièvre 2016) qui vise à construire un savoir pour l'action, à destination des praticiens, en combinant des connaissances expérientielles et scientifiques. Cette recherche s'inscrit dans le «practice turn» (Schatzki, Knorr Cetina \& Savigny 2001) qui se propose d'investir ce que font les acteurs et comment ils le font en situation ordinaire à l'écart des théories surplombantes de l'action rationnelle. Depuis le xviII siècle, les équipes des expéditions polaires construisent des connaissances et développent des pratiques leur permettant de mener à bien des projets d'innovation d'exploration. Nous cherchons donc à apprendre de ces savants ordinaires, à approcher les connaissances indigènes, mais aussi à les mettre en perspective avec des savoirs scientifiques de diverses disciplines pour produire des savoirs pour l'action en mesure de contribuer à la réussite des projets d'expédition.

La question alimentaire est centrale pour les expéditeurs polaires; elle conditionne en grande partie la réussite ou l'échec de l'expédition. La quantité de nourriture détermine en partie la durée de l'expédition, elle impacte directement le poids transporté et l'effort à fournir tandis que l'effort fourni ne peut être indépendant de l'alimentation. Concevoir une «bonne» alimentation apparaît dès lors essentiel. Les différentes enquêtes que nous avons menées montrent tout d'abord que la conception d'une «bonne» alimentation pour une expédition polaire à ski est traversée par différentes 
logiques complémentaires mais également en tension. Il s'agit d'une logique nutritionniste supposant de calculer les nutriments nécessaires à l'effort physique consenti, d'une logique de contrôle du poids et du volume de nourriture et enfin d'une logique de goût. L'identification de ces logiques constitue un premier savoir pour l'action des praticiens afin d'élaborer leurs propres alimentations (Lièvre 2004). En étudiant les pratiques des expéditeurs polaires, nous formalisons différents «styles» d'expédition, différentes manières de concevoir et de mettre en œuvre une expédition polaire (Lièvre, Rix-Lièvre \& Recopé 2003). Chaque style révèle une cohérence et une orientation définissant ce qui est «bon» ou «mauvais» (Canguilhem 2007) dans la conception et la mise en ouvre de l'expédition. La stratégie alimentaire n'échappe pas à cette orientation. La formalisation de différents styles d'expédition fournit un autre outil pour les praticiens permettant de mettre en perspective leurs propres arbitrages.

\section{En ligne}

Retrouver l'article complet sur journals.openedition.org/tc: Techniques\&Culture 69 «Le temps des aliments».

\section{Les auteurs}

Pascal Lièvre, professeur agrégé des universités en sciences de gestion, Université Clermont Auvergne, CRCGM EA 3849, développe un programme de recherche autour du management des situations extrêmes.

Géraldine Rix-Lièvre, professeure des universités en STAPS à l'Université Clermont Auvergne, Laboratoire ACTé EA 4281, développe une anthropologie des pratiques corporelles centrée sur les fondements cognitifs et sensibles de l'expérience.

Michel Récopé, maître de conférences HDR en STAPS à l'Université Clermont Auvergne, Laboratoire ACTé EA 4281, s'intéresse aux fondements sensibles de l'expérience et de l'activité.

\section{Iconographie}

Image d'ouverture. Préparation de la pulka avant le départ en bateau, expédition Norvège 2005. @ P. Lièvre.

$1 \& 2$. (C) G. Rix-Lièvre.

\section{Références}

Canguilhem, G. 2007 [1966] Le normal et le pathologique. Paris: P.U.F

Lièvre, P., Récopé, M. \& G. Rix-Lièvre 2003 «Finalités des expéditeurs polaires et principes d'organisation » in P. Lièvre dir. Logistique des expéditions polaires à ski. Paris: GNGL: 85-101.
Lièvre, P. 2004 «Vers un savoir d'action en sciences de gestion. Le cas des expéditions polaires », Gérer et Comprendre 75 : 4-17

- 2016 «État et développement d'un programme de recherche. Management des Situations Extrêmes», Revue Française de Gestion 257: 79-94.

Schatzki, T., Knorr Cetina, K. \& E. Savigny 2001 The Practice Turn in Contemporary Theory. New York: Routledge.

\section{Pour citer l'article}

Lièvre, P., Rix-Lièvre, G. \& M. Récopé 2018 «Concevoir une bonne alimentation. Les pratiques alimentaires des expéditeurs polaires», TechniquesE Culture 69 «Le temps des aliments», p. 104-107. 